\title{
Application of $\mathrm{X}$-ray resonant diffraction to structural studies of liquid crystals
}

\author{
P. Barois ${ }^{1, a}$, H. Gleeson ${ }^{2}$, C.C. Huang ${ }^{3}$, and R. Pindak ${ }^{4}$ \\ 1 Centre de Recherche Paul Pascal, Université de Bordeaux, 33600 Pessac, France \\ 2 School of Physics \& Astronomy, University of Manchester, M13 9PL Lancs, UK \\ 3 School of Physics and Astronomy, University of Minnesota, Minneapolis, MN 55455, USA \\ 4 Photon Sciences Directorate, Brookhaven National Laboratory, Upton, NY 11973, USA
}

Received 6 January 2012 / Received in final form 23 March 2012

Published online 15 June 2012

\begin{abstract}
Liquid crystals are soft materials that combine the fluidity of disordered liquids and the long range orientational or positional order of crystalline solids along one or two directions of space. X-ray scattering is widely and generally successfully used to investigate and characterize the microscopic structure of most liquid crystals. In many cases however, the Bragg reflections are forbidden by special symmetries of the unit cell and the low dimensional structure of the liquid crystalline phases are out of reach of conventional X-ray experiments. We show in this paper that this problem can be overcome by resonant scattering of X-rays as it reveals the anisotropy of the tensor structure factor. We review various examples in which the restored forbidden reflections reveal unambiguously the hidden structure of liquid crystalline phases. Moreover, we show that in some cases, a fine analysis of the polarization of the Bragg reflections enables one to discriminate between different structural models. These studies solved long standing questions about biaxial liquid crystal structures and provided new insights into physical phenomena such as supercritical behaviour or commensurate-incommensurate transitions.
\end{abstract}

\section{Introduction: Liquid crystals}

Liquid crystals (LC) or mesophases are intermediate states of condensed matter which combine long range positional or orientational order along some directions of space (like in a crystal) and liquid-like disorder along other directions [1]. The most common liquid crystalline phases are obtained with organic molecules or in solutions of surfactants. In the first case, the phase transitions are triggered by changing the temperatures (so-called thermotropic LC) whereas the structures are controlled by the fraction of solvent in the second case (lyotropic LC). Only thermotropic liquid crystals will be considered in this paper. The liquid crystalline properties are mostly due to the anisotropic shape of the molecules (or mesogens). Rods and disks are the most

\footnotetext{
a e-mail: barois@crpp-bordeaux.cnrs.fr
} 


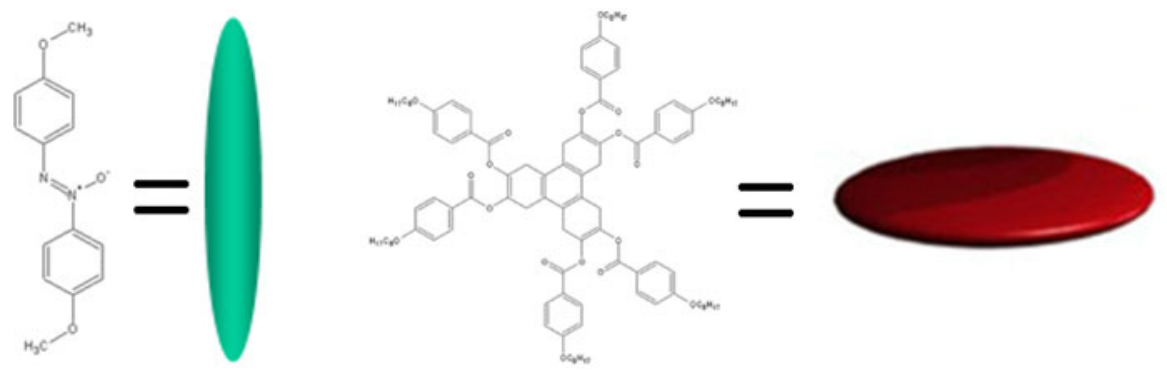

Fig. 1. Examples of rod like (left) and disk like (right) mesogens.

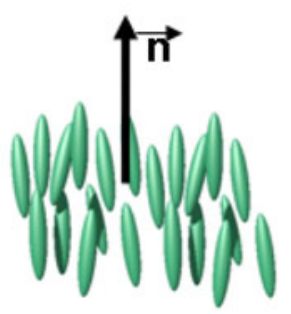

(a)

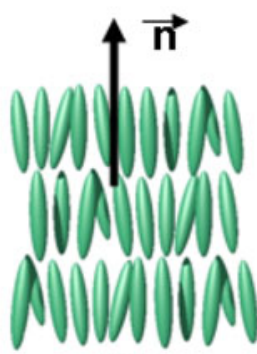

(b)

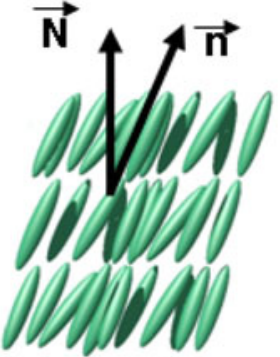

(c)

\section{Nematic}

Smectic A

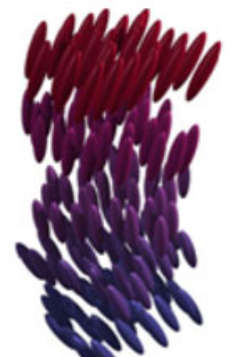

(d)

Cholecteric

Fig. 2. Schematic views of the Nematic (a) the Smectic A (b) the Smectic C (c) and the Cholesteric (d) phases. $\mathbf{n}$ is the nematic diretor. $\mathbf{N}$ is the layer normal in smectic phases.

familiar shapes. Figure 1 shows examples of calamitic (or rod-like) and discotic liquid crystals.

The most common liquid crystalline phases are the nematic, in which molecules line up along a preferred direction denoted by a unit vector $\mathbf{n}$ (the director). In a nematic phase, the centres of mass of the molecules are distributed in space with no long range positional correlations, like in conventional liquids. Nematics actually can flow like liquids. However, they exhibit long range orientational correlations which result in strong anisotropy in various physical properties. The combination of a strong birefringence and a fluid state is the basis of the well known applications of nematics in liquid crystal displays (LCD).

A more ordered mesophase called "smectic" is often obtained upon cooling a nematic of rods. In a smectic phase, the organic molecules are distributed in layers with no long range order in their plane. The layers are regularly stacked along their normal vector $\mathbf{N}$. The smectic phases hence combine the long range orientational order of nematics, plus a one-dimensional positional order along the layer normal, plus a liquid like disorder within the plane of the layers. A uniaxial smectic with the director $\mathbf{n}$ parallel to the layer normal $\mathbf{N}$ is called smectic A $(\mathrm{SmA})$, whereas the two vectors make a finite angle $\theta$ in the smectic $\mathrm{C}$ phase $(\mathrm{SmC})$.

More complex mesophases with a higher degree of order exist. In particular, two dimensional arrays of fluid columns are often found in disk-like systems.

An interesting and quite common feature of thermotropic liquid crystal is molecular chirality which may arise from the presence of an asymmetric carbon. A common consequence is the formation of twisted structures. Two examples are famous: (i) the chiral nematic, also known as cholesteric (Fig. 2(d)), exhibits a helical structure of the 

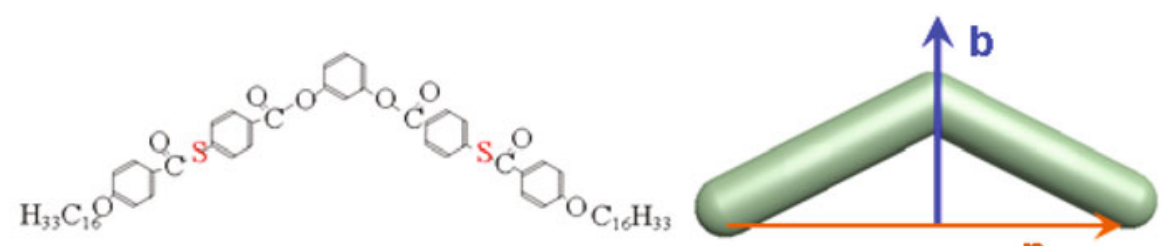

n

Fig. 3. Left: example of bent-core molecule $(16 \mathrm{OTBB})_{2}$. Right: the convenient picture of a "bow" is used to identify the string $\mathbf{n}$ and the arrow $\mathbf{b}$.

director with a well defined pitch. Selective reflection of light occurs when the pitch is in the optical range. (ii) the chiral smectic $\mathrm{C}$ phase $\left(\mathrm{SmC}^{*}\right)$ exhibits ferroelectricity in the plane of each layer along a direction $\mathbf{n} \times \mathbf{N}$ as a result of the chiral symmetry breaking. In addition, a helical precession of the local polarization is observed along the layer normal $\mathbf{N}$. More than 10 years after the discovery of the Sm-C* phase, it was recognized that their existed a rich variety of Sm-C* sub-phases with different types of interlayer molecular orientational order and associated electrical polarization responses [1] - some of special interest for device applications. The resonant scattering technique provided a direct determination of the structure of these $\mathrm{Sm}-\mathrm{C}^{*}$ sub-phases, their phase transitions, and electric-field response [2]. Section 2 reviews the most significant results, up to the most recent.

In recent years, a new type of mesogens has attracted a lot of interest, the socalled bent-core molecules. Unlike rods and disks, the description of the orientation of a bent-core molecule requires two vector fields. If the molecule is viewed like a bow, the two "directors" are the string and the arrow (Fig. 3). The phases resulting from the spatial organization of these two fields have been assigned names $B_{1}$ to $B_{7}$ as they were discovered [3]. The application of resonant scattering to the elucidation of the microscopic structures of the $\mathrm{B}_{2}$ phase will be detailed in section 3 .

\section{Antiferroelectric smectic liquid crystals and related sub-phases}

The discovery in 1989 of the antiferroelectric, chiral smectic $\mathrm{C}$ phase $\left(\mathrm{SmC}_{\mathrm{A}}^{*}\right)$ in the MHPOBC liquid crystal compound [4] started an intense research effort to clarify the properties and origin of this novel structure. One result of this work was the discovery of other smectic C sub-phases, including the so-called $\mathrm{SmC}_{\alpha}^{*}$ and the intermediate $\mathrm{SmC}_{\mathrm{FI} 1}^{*}$ and $\mathrm{SmC}_{\mathrm{FI} 2}^{*}$ phases. Upon decreasing temperature, the typical phase sequence of mesogens exhibiting the antiferroelectric phase would appear as $\mathrm{SmC}_{\alpha}^{*}, \mathrm{SmC}^{*}$, $\mathrm{SmC}_{\mathrm{FI} 2}^{*}, \mathrm{SmC}_{\mathrm{FI} 1}^{*}$ and $\mathrm{SmC}_{\mathrm{A}}^{*}[5]$.

Only the structures of the classical $\mathrm{SmC}^{*}$ and of the antiferroelectric $\mathrm{SmC}_{\mathrm{A}}^{*}$ phases (Fig. 4) were fully understood [6]. Many different experimental techniques have been applied during a decade in order to characterize the other smectic $\mathrm{C}$ sub-phases, but none of these methods have been able to provide microscopic pictures of these phases. In particular X-ray diffraction, which is normally a powerful tool to resolve these issues, could not discern any difference between the various sub-phases. The fundamental reason for this is illustrated in Fig. 4 in which the microscopic structures of the $\mathrm{SmC}^{*}$ and $\mathrm{SmC}_{\mathrm{A}}^{*}$ phases are shown. The unit cell of the $\mathrm{SmC}$ phase is one layer of thickness $\mathrm{d}$ and conventional diffraction usually reveals a series of Bragg reflections at wave vectors $Q_{\mathrm{o}}=2 \pi / \mathrm{d}, 2 \mathrm{Q}_{\mathrm{o}}, 3 \mathrm{Q}_{\mathrm{o}} \ldots$ along the layer normal axis $\mathrm{z}$. The unit cell of the $\mathrm{SmC}_{\mathrm{A}}^{*}$ phase is two layers, and the fundamental reciprocal vector is hence $\mathrm{Q}_{\mathrm{o}} / 2$. Unfortunately, the odd reflections are forbidden by the particular symmetry of the unit cell (i.e. $\mathrm{x}-\mathrm{y}$ glide plane or $2_{1}$ screw axis). Consequently, the two phases $\mathrm{SmC}$ 


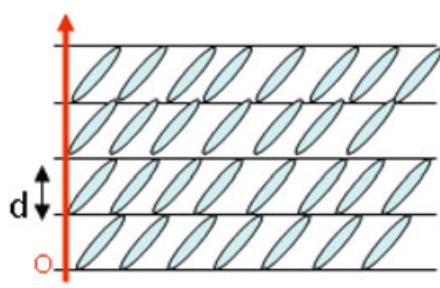

(a)

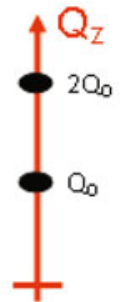

(b)

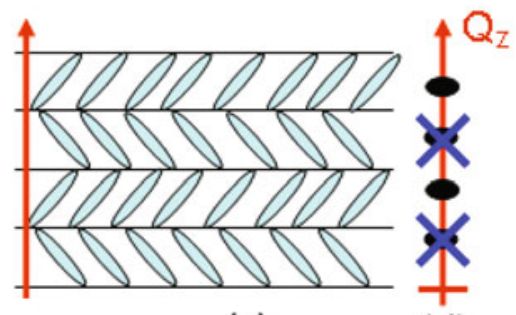

(c)

(d)

Fig. 4. Microscopic structure of the $\mathrm{SmC}$ (a) and the $\mathrm{SmC}_{\mathrm{A}}^{*}$ (c) phases. The corresponding reciprocal spaces are shown in (b) and (d). The odd reflections are forbidden in (d) by the $2_{1}$ screw axis symmetry element (for chiral molecules) or glide plane symmetry (for racemic mixtures or achiral molecules).

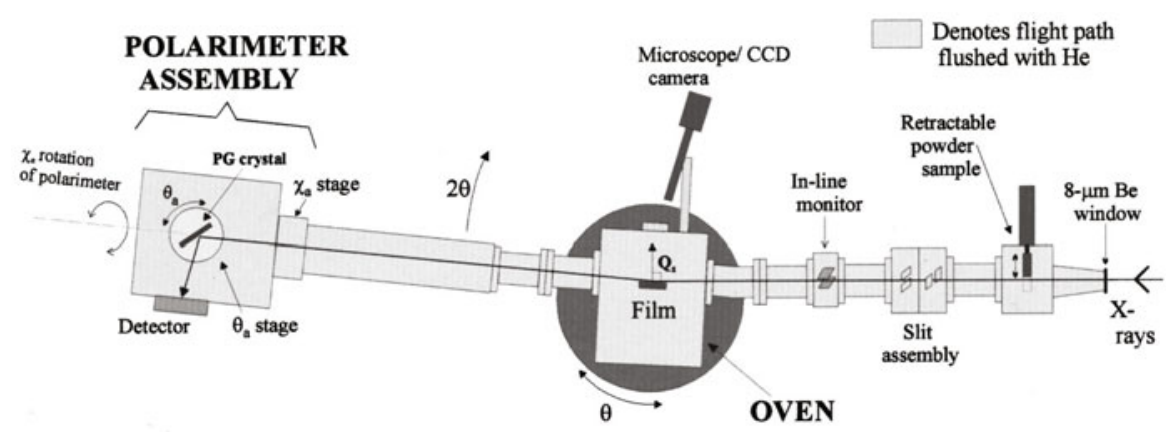

Fig. 5. Schematic diagram of the dedicated experimental setup at beamline X19A of the National Synchrotron Light Source, Brookhaven National Laboratory, USA [8]. The free standing smectic film is prepared in a two-stage oven. A pyrolytic graphite crystal is used to analyse the polarization of the resonant Bragg peaks. It can be removed to collect all the polarization components along the $2 \theta$ direction. The retractable powder sample is used to determine the absorption edge by collecting the fluorescence signal with an additional detector (not shown).

and $\mathrm{SmC}_{\mathrm{A}}^{*}$ are undistinguishable by $\mathrm{X}$-ray diffraction. The condition for forbidden reflection holds for all the other sub-phases and conventional X-ray scattering only revealed the basic layer unit $\mathrm{d}$.

The solution came from resonant X-ray scattering performed at sulphur K-edge on the $\mathrm{n}=10$ member (hereafter referred to as 10OTBBB1M7) of the homologous chiral LC series whose molecular structure is given in Fig. 6 [7]. The bulk 10OTBBB1M7 enantiomer shows the following phase sequence: Isotropic $\left(152.6^{\circ} \mathrm{C}\right) \mathrm{SmA}\left(123.6^{\circ} \mathrm{C}\right)$ $\mathrm{SmC}_{\alpha}^{*}\left(120.2^{\circ} \mathrm{C}\right) \mathrm{SmC}^{*}\left(119.2^{\circ} \mathrm{C}\right) \mathrm{SmC}_{\mathrm{FI} 2}^{*}\left(114.0^{\circ} \mathrm{C}\right) \mathrm{SmC}_{\mathrm{FI} 1}^{*}\left(112.0^{\circ} \mathrm{C}\right) \mathrm{SmC}_{\mathrm{A}}^{*}$ $\left(109.7^{\circ} \mathrm{C}\right)$ crystal. The experiments were conducted at beamline X19A of the National Synchrotron Light Source (Fig. 5). The smectic liquid crystalline samples were prepared as free standing films, about 1 micrometer thick. This geometry guarantees an excellent alignment of the smectic layers parallel to the surface of the film. The orientation of the LC molecules within each layer can be described by a sequence of azimuthal angles $\Psi_{i}$ along the $\mathrm{z}$ axis (Fig. 7). The diffraction data were collected in the Bragg geometry by means of a three-circle goniometer. The energy of the beam was tuned at the maximum of the fluorescence energy scan at $2475 \mathrm{eV}$ (Fig. 6) in order to reveal the forbidden reflections. The whole flight path was continuously flushed with helium to minimize absorption of the low energy X-ray photons. The X-ray intensity 


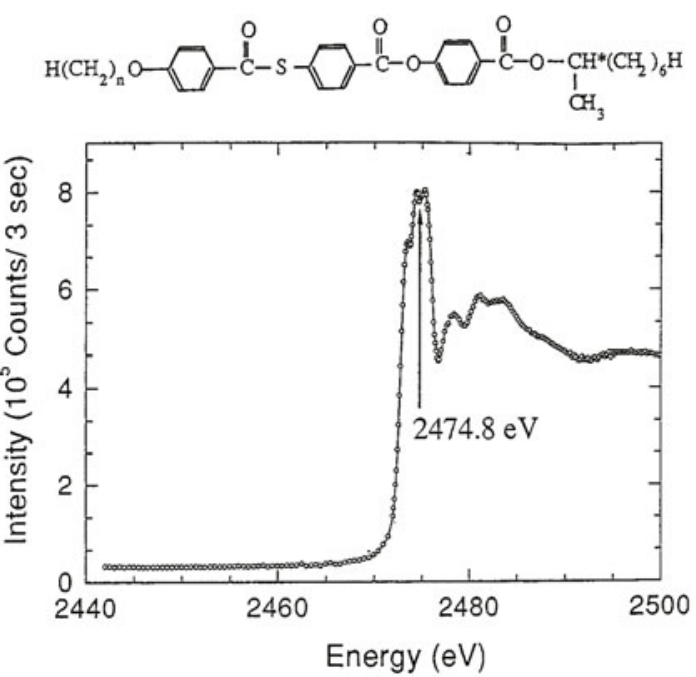

Fig. 6. Top: homologous series of which 10OTBBB1M7 is the $\mathrm{n}=10$ member. Bottom: Fluorescence intensity versus X-ray beam energy incident onto bulk 10OTBBB1M7 powder sample 4 (from ref. [7]).

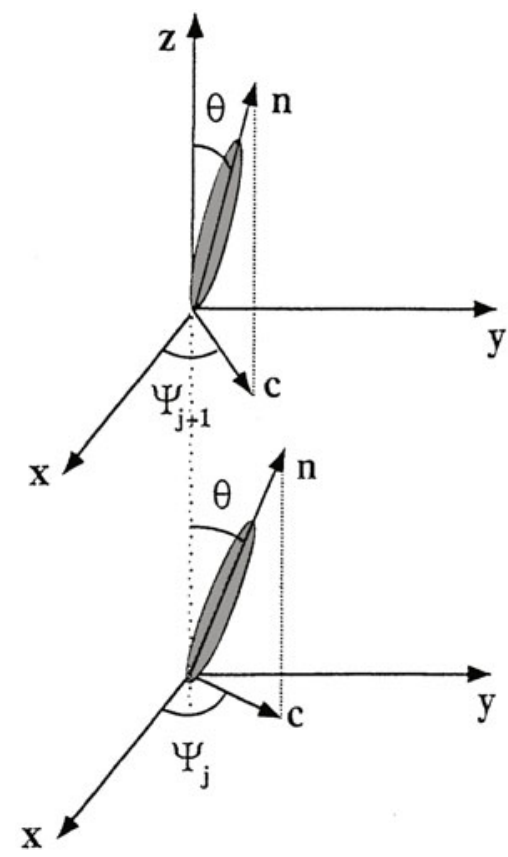

Fig. 7. Schematic diagram illustrating the orientation of molecules in neighbouring SmCtype layers. Tilt angle $\theta$ in layers is the same, while azimuthal angles $\Psi$ differ (from ref. [7]).

scans are shown in Fig. 8 in reduced $Q_{z} / Q_{0}$ units (with $Q_{0}=2 \pi / d$ ). In addition to the usual non resonant ( $\left.\begin{array}{lll}0 & 0 & 2\end{array}\right)$ Bragg peaks, a series of resonant features are observed. The simplest case is the achiral $\mathrm{SmC}_{\mathrm{A}}$ phase of the racemic mixture (Fig. 8(e)). Sharp half order reflections are detected in $\left(\begin{array}{lll}0 & 0 & 3 / 2\end{array}\right)$ and $(005 / 2)$ positions, hence revealing the two layer unit cell drawn in Figure 4c. In the chiral case of the pure enantiomer (Fig. 8(d)), a long pitch helical precession develops along the $\mathrm{z}$ axis in the $\mathrm{SmC}_{\mathrm{A}}^{*}$ 


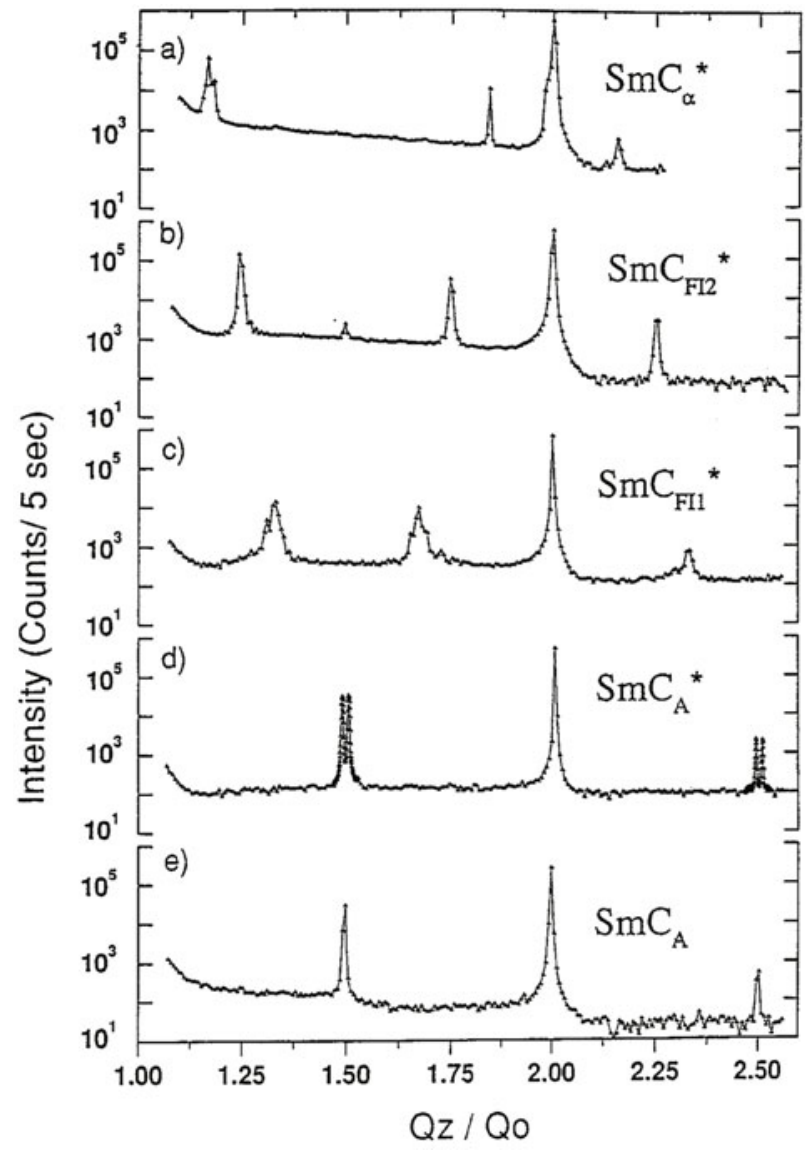

Fig. 8. X-ray intensity scans in the indicated phases of (R)-enantiomer (plots a-d) and racemic 10OTBBB1M7 (plot e). Temperature is lowered from a to d. The polarimeter was not used in these scans (from ref. [7]).

phase. Consequently, the helical period is slightly different from 2 layers and the resonant Bragg peaks are slightly off the half order positions. The splitting of the resonant signal gives the value of the helical pitch (125 layers or $480 \mathrm{~nm}$ in Fig. 8(d)) but not its sign. At higher temperature in the $\mathrm{SmC}_{\mathrm{FI} 1}^{*}$ phase, the resonant peaks show up at $4 / 3,5 / 3$ and $7 / 3$ positions, hence revealing a 3-layer superlattice. The unit cell grows to four layers in $\mathrm{SmC}_{\mathrm{FI} 2}^{*}$ phase as the resonant peaks move to quarter order positions. Finally, in the $\mathrm{SmC} \alpha^{*}$ phase, satellite resonant peaks appear at incommensurate positions which denote a helical arrangement of the molecules with a variable pitch roughly evolving from five to eight layers upon heating.

Figure 9 shows the variation of the intensity of the Bragg peaks as a function of the energy of the incident X-ray beam in the $\mathrm{SmC}_{\mathrm{FI} 2}^{*}$ phase. The intensity of the quarter order peaks vanishes as the energy is lowered, whereas the intensity of the non resonant ( $\left.\begin{array}{lll}0 & 0 & 2\end{array}\right)$ Bragg peak remains constant, hence proving the resonant nature of the non integer Bragg peaks.

Interpretation of data: The restoration of forbidden reflections by resonant scattering was first evidenced by D.H Templeton and L.K Templeton [9]. Let us recall briefly that resonant X-ray scattering occurs when the energy of the X-ray radiation approaches the values required to excite an inner-shell electron into an empty state of 


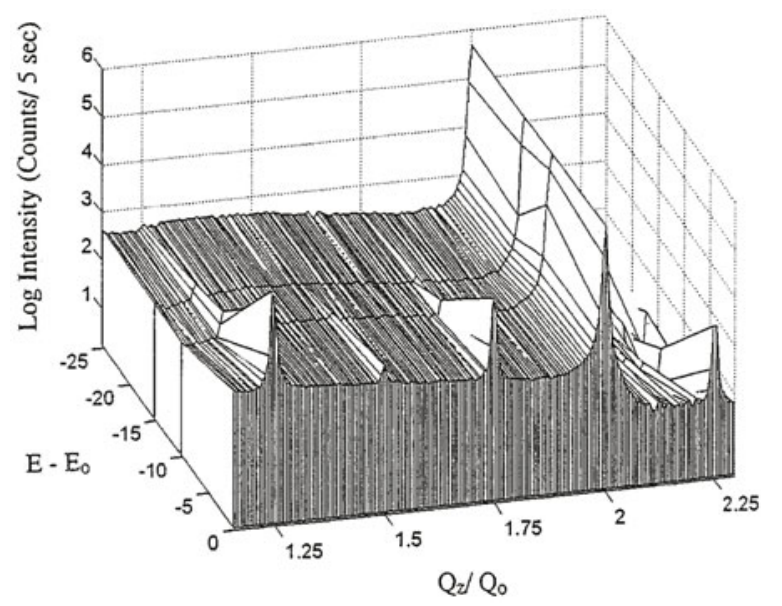

Fig. 9. X-ray intensity in the $\mathrm{SmC}_{\mathrm{F} 12}^{*}$ phase of the 10OTBBB1M7 (R)-enantiomer as a function of the incident beam energy relative to the sulfur K-edge absorption maximum (from ref. [7]).

the outer shell [10]. In such circumstances, the atomic scattering factor $f a$ exhibits a complex frequency-dependent contribution in addition to the classical (non-resonant) term $f_{0}$, which is the Fourier transform of the electron density. The resonant contribution depends on the atom and becomes substantial near its absorption edges. Because of the local anisotropy of the outer-shell unoccupied states, the resonant structure factor is a tensor that reflects the symmetry of the resonant atom within the molecule. The anisotropy of this tensor leads to the observation of forbidden reflections and determines their nontrivial polarization properties. The variations of the polarization state and of the intensity of the resonant forbidden reflections have been worked out by V.E. Dmitrienko as a function of the rotation angle $\varphi$ of the sample about the scattering vector $\mathbf{Q}_{\mathbf{z}}$ for cubic crystals in kinematic theory [11]. This theoretical work was adapted to the problem of antiferroelectric smectics by A.-M. Levelut and B. Pansu [12]. Two types of models have been considered in [12] namely the clock model in which the orientation of the molecules form a regular helix of pitch $\mathrm{P}=2 \pi \mathrm{d} /\left(\Psi_{\mathrm{i}+1}-\Psi_{\mathrm{i}}\right)$ along $\mathrm{z}$ and the Ising model $[4,5]$ in which the difference between azimuthal angles of adjacent layers $\left(\Psi_{\mathrm{i}+1}-\Psi_{\mathrm{i}}\right)$ can take only two values 0 or $\pi$.

The clock model generates two and only two sets of satellite peaks of splitting $\Delta=\mathrm{d} / \mathrm{P}$ and $2 \Delta$ (in $\mathrm{Q}_{0}$ units) and different polarizations. The satellites may be simply indexed by a notation $(1, \mathrm{~m})$ in which $\mathrm{l}$ is the index of the non resonant peak, and $\mathrm{m}( \pm 1, \pm 2)$ is the index of a satellite. They are located at positions $\mathrm{Q}_{\mathrm{z}}$ satisfying

$$
\frac{Q_{z}}{Q_{0}}=l+m d / P
$$

Figure 10 summarizes the profile of the resonant satellite peaks as calculated from theory [12] and measured experimentally [8] in the well known helical $\mathrm{SmC}^{*}$ phase. The agreement is excellent.

The clock and Ising models actually both generate 3-layer, 4-layer or incommensurate superlattices. Their polarization properties are however quite different. In the 4-layer $\mathrm{SmC}_{\mathrm{FI} 2}^{*}$ phase for instance, and for incident $\sigma$ polarization, the $(1, \pm 2)$ peaks are either nil or $\pi$ polarized in the Ising models, whereas they are $\sigma$ polarized in the clock model. Figure 11 shows a series of polarimeter scans that clearly demonstrate that the $(1,1)$ and $(2,-1)$ satellites are $\pi$ polarized, whereas the $(1,2) /(2,-2)$ signal is $\sigma$ polarized, hence ruling out the Ising models [8]. 

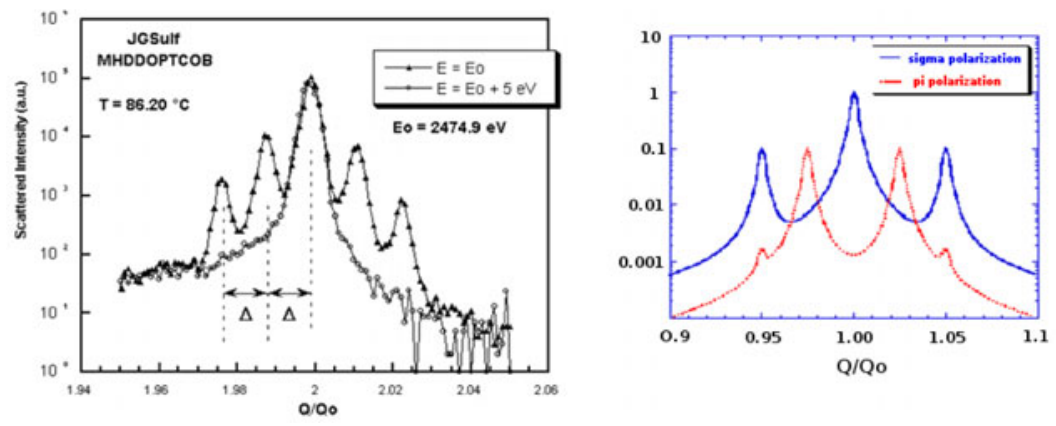

Fig. 10. Left: intensity profile of the pair of resonant satellite peaks recorded in the helical $\mathrm{SmC}^{*}$ phase. The helical pitch $1 / \Delta$ is 87 layers. The four satellite peaks are indexed $(2,-2)$, $(2,-1),(2,1)$ and $(2,2)$ from left to right. Right: theoretical profile calculated for the clock model for at $\sigma$ golarization of the incident beam.
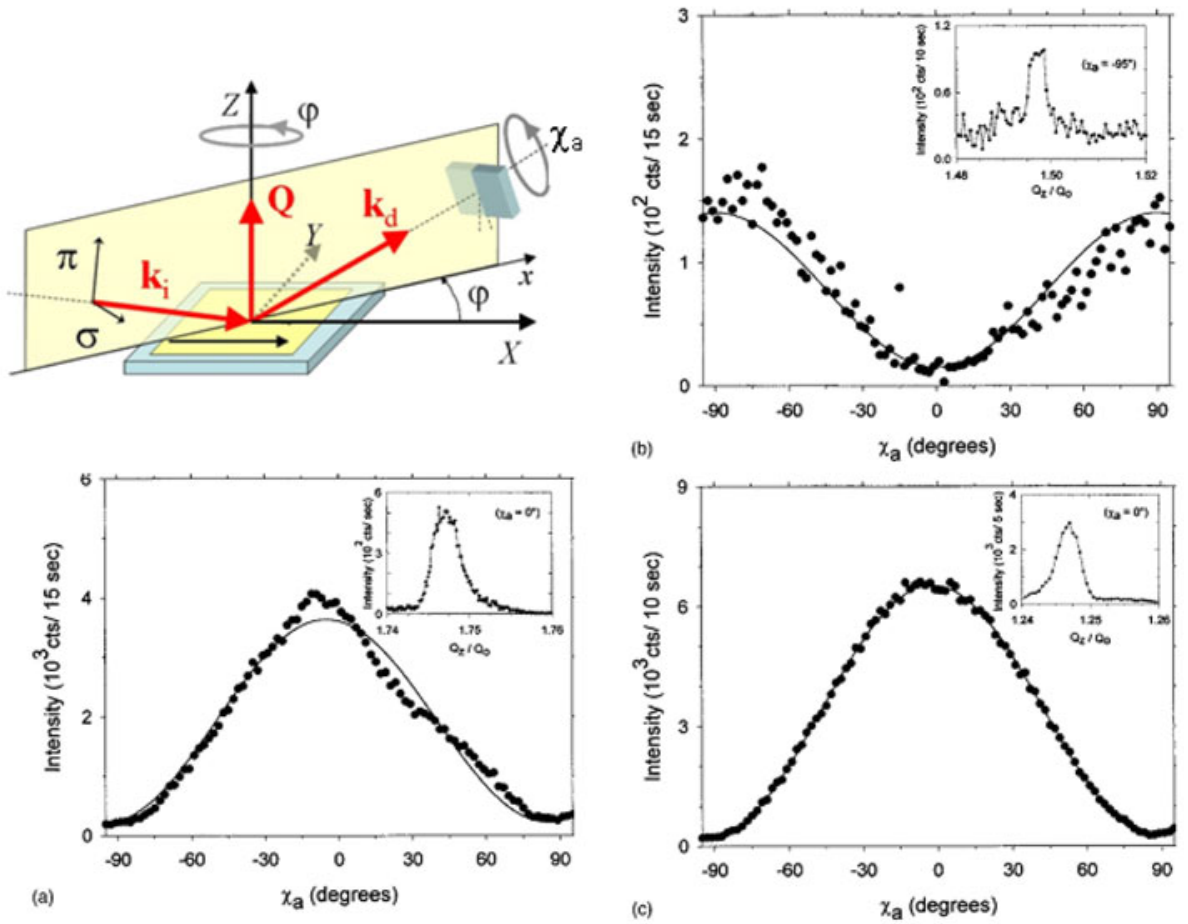

Fig. 11. Top left: scattering geometry defining the polarization vectors. The analyzer is a graphite crystal that has a Bragg angle of 48.3 degrees and a residual intensity of $3.5 \%$ at extinction. The azimuthal rotation $\varphi$ will be used in section 3. (a,b,c) Polarization scans recorded in the 4-layer $\mathrm{SmC}_{\mathrm{FI} 2}^{*}$ phase. A maximum at $\chi_{a}=0$ corresponds to a $\pi$ polarization for the $(2,-1)$ (a) and $(1,1)$ (c) satellites. A minimum at at $\chi_{a}=0$ corresponds to a $\sigma$ polarization for the $(1,2) /(2,-2)$ signal (b) (from ref. [8]).

The polarization of the resonant peaks was likewise checked in all the phases and found to always rule out the Ising model, lending support to the clock model.

The optical properties were however not in good agreement with the clock model. In particular, the strong optical anisotropy currently observed in the intermediate phases can be understood in the framework of the Ising models, but not in the clock 

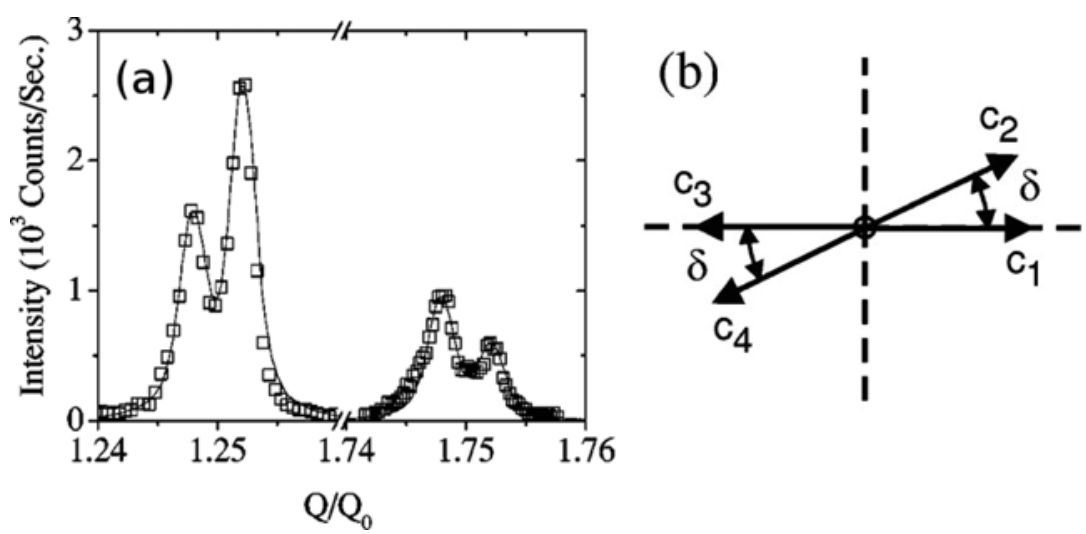

Fig. 12. (a) High resolution $Q_{z}$ scans across the resonant quarter order reflections in the 4-layer $\mathrm{SmC}_{\mathrm{FI} 2}^{*}$ phase. The solid line is a fit to the distorted clock model shown in (b) with a distortion angle $\delta=15$ degrees (from ref. [13]).

structure. In order to solve this apparent contradiction, high resolution resonant scattering studies were carried out [13].

These new studies showed that the quarter order reflections in the $\mathrm{SmC}_{\mathrm{FI} 2}^{*}$ phase were in fact split (Fig. 12(a)). This splitting is not consistent with the regular clock model described in [12]. A distorted clock model was proposed instead [13]. In this new model, the rotation angle of the tilt direction $\left(\Psi_{i+1}-\Psi_{i}\right)$ is no longer uniform but alternatively takes two values $\delta$ and $\pi-\delta$ as shown in Fig. 12(b). The values of $\delta$ extracted from the fits (Fig. 12(a)) range from 15 to 20 degrees, depending on temperature. The variations of the resonant scattering profiles as a function of the distortion of the clock models are calculated in [14].

The structure of the 3-layer $\mathrm{SmC}_{\mathrm{FI} 1}^{*}$ phase was likewise studied in detail. The splitting of the third-order resonant reflections revealed a distorted clock structure in which the basic azimuthal rotations are $\delta \approx 55$ degrees, and $(\pi-\delta / 2)$ instead of $2 \pi / 3[15]$.

In both cases, the basic 4-layer and 3-layer cells depicted above undergo a long pitch precession along $\mathrm{z}$. These new pictures, summarized in Fig. 13 [16], reconcile the resonant X-ray scattering and the optical experiments. Indeed, the basic 4-layer unit cell of the distorted structure shown in Fig. 12(b) is strongly biaxial and consistent with the observed strong optical anisotropy in ellipsometric signal [17].

On the contrary, no deviation from the regular clock model was detected in the $\mathrm{SmC} \alpha^{*}$ phase, which suggests that the incommensurate helical pitch of this phase is equivalent to the classical $\mathrm{SmC}^{*}$ helix. This intriguing similarity was investigated in reference [18]. It was found that a continuous transition can occur between the two phases, hence confirming the equivalence of their symmetries. Moreover, a first order $\mathrm{SmC}^{*}-\mathrm{SmC}_{\alpha}^{*}$ transition terminating on a critical point was found in a binary mixture [19].

Resonant scattering at higher energies. Although the technique of resonant scattering has proved to be very efficient to resolve the unknown structures of chiral smectic liquid crystals, the first experiments performed at the sulphur K-edge were limited to liquid crystal molecules possessing the sulphur element and to sample geometries with a free open surface. Indeed, the low energy X-ray beam at $2.47 \mathrm{keV}$ is completely absorbed by any kind of container wall. This last limitation was overcome by the synthesis of novel liquid crystal molecules in which the resonant atom is selenium or bromine. The higher energy corresponding to the absorption K-edge of 


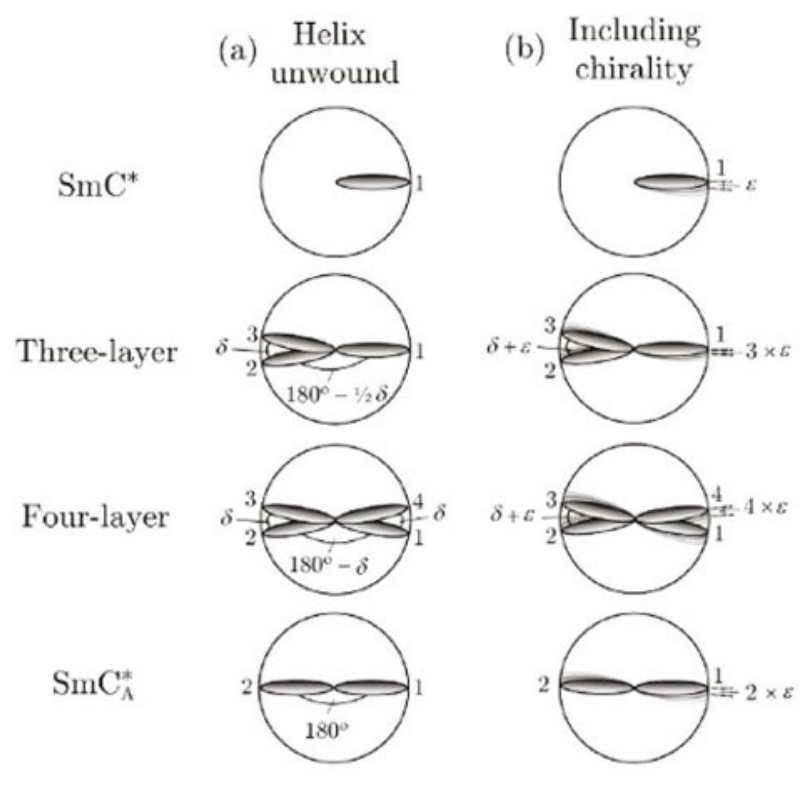

( Layer normal

Fig. 13. Summary of the microscopic structures of the smectic C sub-phases as demonstrated by resonant scattering of X-rays. The Figures represent top views of the unit cells constituted of 1, 3, 4 and 2 layers from top to bottom. The structure of the $\mathrm{SmC}_{\alpha}^{*}$ phase (not shown) is equivalent to the $\mathrm{SmC}^{*}$ phase (top) with a larger twist $\varepsilon$ though (from ref. [16]).

these heavier elements, close to $13 \mathrm{keV}$, enables the use of a device geometry with the LC contained between thin glass plates with transparent electrodes.

Resonant scattering performed on chiral liquid crystals at the bromine K-edge $(13.49 \mathrm{keV})$ was however disappointing as the resonant signal turned out to be very low, even in the favourable geometry of free standing film [14].

Experiments performed at selenium K-edge $(12.66 \mathrm{keV})$ were much more successful. In this case, the strong resonant signal enables resonant scattering experiments in liquid crystal devices. In this geometry, a liquid crystal sample (15 to $30 \mu \mathrm{m}$ thick) is enclosed between two glass plates. A conductive ITO layer enables the application of an electric field. A surface treatment is used to align the smectic layers perpendicular to the glass plates in the so-called "bookshelf" geometry (Fig. 14(a)). In the tilted smectic $\mathrm{C}$ phases, the bookshelf arrangement generally breaks up to form a chevron structure with an angle $\theta_{c}$ close to the smectic C tilt (Fig. 14(b)). Figure 14(c) shows an example of a liquid crystal molecule having a selenium atom within a selenophene ring [20,21]. The bulk phase sequence comprises the antiferroelectric $\mathrm{SmC}_{\mathrm{A}}^{*}$ and the ferrielectric $\mathrm{SmC}_{\mathrm{FI} 1}^{*}$ and antiferroelectric $\mathrm{SmC}_{\mathrm{FI} 2}^{*}$ subphases. The $\mathrm{SmC}_{\mathrm{A}}^{*}$ and the 4layer $\mathrm{SmC}_{\mathrm{FI} 2}^{*}$ phases could be characterized by the detection of their resonant peaks in the device. Moreover, the transformation of the structures under application of an electric field could be monitored.

Two regimes shown were identified. At low field, the resonant signal decays continuously hence revealing the progressive unwinding of the antiferro or ferrielectric structure. Above a field threshold, the chevron structure abruptly switches to the bookshelf alignment and the resonant signal vanishes, indicative of the unwound ferroelectric smectic C structure. The discovery of materials with remarkably wide intermediate $\mathrm{SmC}_{\mathrm{FI} 1}^{*}$ and $\mathrm{SmC}_{\mathrm{FI}}^{*}$ phases [22] allowed detailed experiments to be performed which 


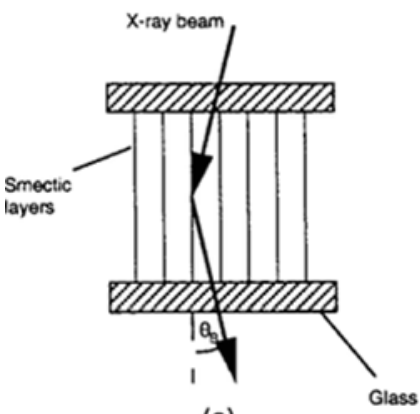

(a)

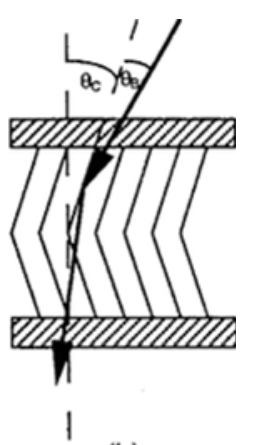

(b)

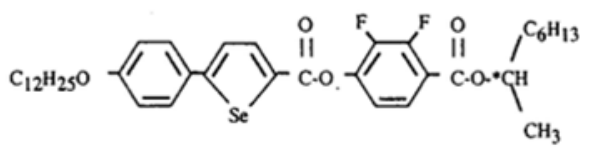

Fig. 14. Top: schematic view of the scattering geometry for a liquid crystal device with (a) a bookshelf layer geometry and (b) a chevron layer geometry. $\theta_{c}$ indicates the chevron angle and $\theta_{B}$ is the Bragg scattering angle. Bottom: example of liquid crystal molecule with a selenium resonant atom. The phase sequence for this material is crystal $\left(46.3^{\circ} \mathrm{C}\right) \mathrm{SmC}_{\mathrm{A}}^{*}$ (82.6) $\mathrm{SmC}_{\mathrm{FI} 1}^{*}$ (83.6) $\mathrm{SmC}_{\mathrm{FI} 2}^{*}$ (86.3) $\mathrm{SmC}^{*}$ (88.2) $\mathrm{SmA}$ (95.8) Isotropic. (from ref. [20]).

deduce the electric field-dependent structures of these systems. A direct transition from the $\mathrm{SmC}_{\mathrm{FI} 2}^{*}$ to either the $\mathrm{SmC}^{*}$ or the $\mathrm{SmC}_{\mathrm{FI} 1}^{*}$ phase [23] is found, showing that the ferrielectric 3-layer structure is relatively stable in a field. Further, a first order field-induced phase transition from the $\mathrm{SmC}_{\mathrm{FI} 1}^{*}$ phase to a new 3-layer ferrielectric phase with a structure in which the angles $\Psi_{I}$ in the unit cell are all $<90^{\circ}$ has been reported [24], and the theoretical description of the threshold offers the potential for determining the inter-layer constants that stabilise the intermediate phases.

Resonant probes in non resonant liquid crystal. Another strong limitation comes from the necessary presence of a resonant element on the liquid crystal molecule. So far, only four elements have been used: sulphur and chlorine with K-edges in the low energy range $(2-3 \mathrm{keV})$, bromine and selenium at higher energies $(12-13 \mathrm{keV})$. Unfortunately, not all liquid crystals of interest possess these elements. This limitation may be circumvented by doping a liquid crystal with a resonant probe. This method was used to investigate the microscopic structure of the subphase of the reference compound MHPOBC [25].

Figure 15 displays the characteristic resonant peaks observed at Selenium K-edge in the antiferro $\mathrm{SmC}_{\mathrm{A}}^{*}$ and 4-layer $\mathrm{SmC}_{\mathrm{FI} 2}^{*}$ phases of $\mathrm{MHPOBC}$ doped with 5 percent in weight of a rod-like molecule possessing a selenium element. Good resonant signals were obtained with concentrations of probe as low as 2 percent. This method opens up the way to structural characterization of all liquid crystals by resonant scattering of X-rays.

Discovery of new LC phases. The novel and spectacular structural results provided by X-ray resonant scattering have guided and stimulated theoretical studies of antiferroelectric smectics [26-30]. The existence of superlattices with periodicities of 2, 3 and 4 layers has been explained by a free energy expansion including the third-nearest neighbours interaction or else between short and long range interactions between layers. Interestingly, some longer periodicities of 5 to 9 layers have been proposed by some advanced models. In reference [30], Hamaneh and Taylor propose the existence of a new phase, with a 6-layer periodicity that had not been observed in experimental systems. As often occurs in liquid crystals physics, binary mixtures of 

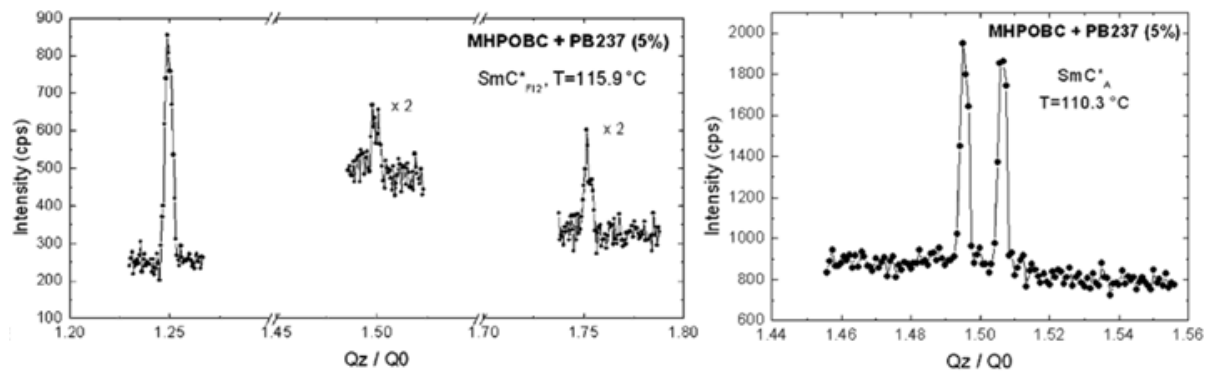

Fig. 15. Resonant signals recorded in the $\mathrm{SmC}_{\mathrm{FI} 2}^{*}$ (left) and the $\mathrm{SmC}_{\mathrm{A}}^{*}$ (right) phases of the MHPOBC liquid crystal doped with $5 \%$ in weight of a resonant probe possessing a selenium atom [25].

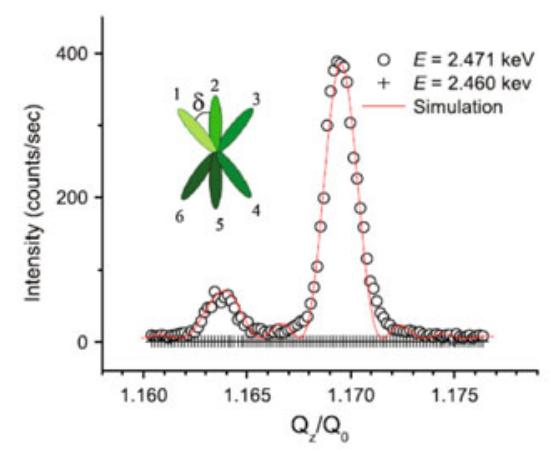

Fig. 16. Resonant Bragg peaks detected in the 6-layer phase. The split peaks centred about position $1+1 / 6$ are fitted to the distorted clock structure shown in inset [31].

mesogens may be used to extend the range of variation of the physical parameters that control the phase behaviour. By using binary mixtures of two chiral smectic mesogens to expand the range of the $\mathrm{SmC}^{*}$ sub-phases, the predicted 6-layer phase was recently discovered [31]. This new phase exhibits a distorted clock structure (Fig. 16) close to but not exactly similar to the Hamaneh-Taylor model. This result confirms that research on chiral antiferroelectric liquid crystals and their subphases is always active and necessary to reach a full understanding of their physics.

Finally, it should be noted that the subphases described in the present paper appear in the litterature with different names, which is somewhat confusing. The 3 and 4-layer subphases in particular were referred to as ferrielectric phases $\left(\mathrm{SmC}_{\mathrm{F} 11}^{*}, \mathrm{SmC}_{\mathrm{FI} 2}^{*}\right)$ or with a subscript $\gamma\left(\mathrm{SmC}_{\gamma 1}^{*}, \mathrm{SmC}_{\gamma 2}^{*}\right)$ or else as $\mathrm{SmC}_{\mathrm{d} 3}^{*}$ and $\mathrm{Sm}_{\mathrm{d} 4}^{*}[27]$. We believe that the latter should be preferred as they clearly refer to the superlattice periodicity and they can be extended to other superlattices like the 6-layer phase $\mathrm{SmC}_{\mathrm{d} 6}$. This clarification is a direct consequence of the results obtained by resonant scattering studies.

\section{Bent-core liquid crystals}

It was shown in the preceding section that the restoration of forbidden reflections by X-ray resonant scattering enabled the unambiguous characterization of the microscopic structure of the chiral liquid crystal $\mathrm{SmC}^{*}$ sub-phases. The polarization properties of the resonant peaks confirmed the interpretations as they perfectly support the model of anisotropy of the tensor susceptibility (ATS) proposed by Dmitrienko, 


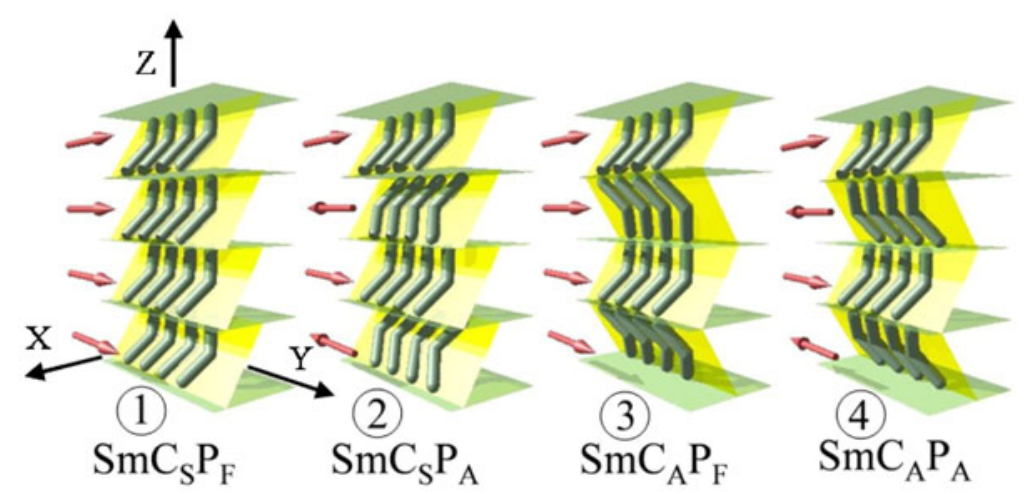

Fig. 17. Sketch of the four possible structures of the $B_{2}$ phase. The arrows show the polarization within each layer. The unit cell is two layers for structures 2,3 , and 4 .

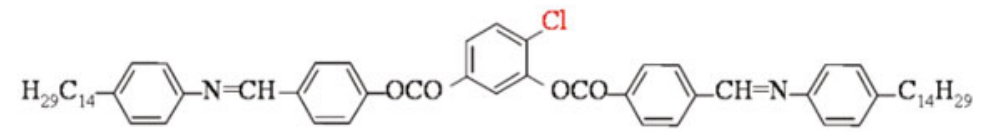

Fig. 18. Molecular structure of the $14 \mathrm{PBCl}$ bent-core liquid crystal. The angle between the two rigid arms of the molecules, of order 120 degrees is not shown here.

Levelut and Pansu [11,12]. These $\mathrm{Sm}^{-\mathrm{C}^{*}}$ sub-phases all exhibit uniaxial symmetry. We will show in this section that in the case of bent-core liquid crystals, which exhibit a biaxial symmetry, a full analysis of the polarization states of the resonant signal is required to elucidate the microscopic structure of the so-called $\mathrm{B}_{2}$ phase.

Early X-ray studies revealed the lamellar and tilted (or smectic $\mathrm{C}$ ) nature of the $\mathrm{B}_{2}$ phase. The bent-core molecules stand on layers of thickness $d$ with no long range in-plane order. The string $\mathbf{n}$ of the bow (Fig. 3) is tilted relative to the layer normal axis $\mathbf{Z}$ wheras the arrow $\mathbf{b}$ points along a direction parallel to the plane of the layers. Four models have been proposed corresponding to the four combinations of the SmC-like tilt of the string (synclinic or anticlinic) and of the arrow polarization

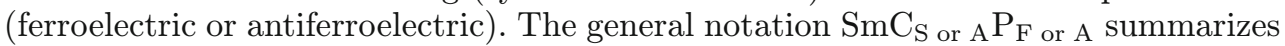
these four states shown in Fig. 17. Link et al. [32] have pointed out that the individual layers of the proposed structures are chiral (i.e., different from their mirror image) although the molecules are achiral. The $\mathrm{SmC}_{\mathrm{S}} \mathrm{P}_{\mathrm{F}}$ and $\mathrm{SmC}_{\mathrm{A}} \mathrm{P}_{\mathrm{A}}$ structures are homochiral whereas the $\mathrm{SmC}_{\mathrm{S}} \mathrm{P}_{\mathrm{A}}$ and $\mathrm{SmC}_{\mathrm{A}} \mathrm{P}_{\mathrm{F}}$ are racemic mixtures of layers of opposite handedness. The in-plane axes $X$ (along the tilt direction) and $Y$ (along the polarization arrow) complete the reference frame as shown in Fig. 17.

The unit cell is one layer for the $\mathrm{SmC}_{\mathrm{S}} \mathrm{P}_{\mathrm{F}}$ structure but two layers of opposite tilt and/or polarization for the other three. In reciprocal space, the basic wave vector component $Q z$ of the latter is then $Q_{0} / 2$, with $Q_{0}=2 \pi / d$. The corresponding half-order Bragg reflections are forbidden [glide plane $(X, Z)$ for the $\mathrm{SmC}_{\mathrm{S}} \mathrm{P}_{\mathrm{A}}$ phase, $(Y, Z)$ for $\mathrm{SmC}_{\mathrm{A}} \mathrm{P}_{\mathrm{F}}$, and $2_{1}$ screw axis for $\left.\mathrm{SmC}_{\mathrm{A}} \mathrm{P}_{\mathrm{A}}\right]$. X-ray resonant scattering is therefore needed to reveal the 2-layer unit cells.

The $\mathrm{B}_{2}$ phases of two bent-core molecules ((16OTBB $)_{2}$ Fig. 3 and 14PBCl Fig. 18) have been investigated by X-ray resonant scattering by A. Cady et al. [33]. These studies revealed the presence of strong resonant Bragg peaks at half-order positions, hence ruling out the homochiral $\mathrm{SmC}_{\mathrm{S}} \mathrm{P}_{\mathrm{F}}$ phase. Moreover, electro-optical studies have evidenced an antiferroelectric behaviour [29,34], and the identification of the structures reduces to distinguishing between the $C_{\mathrm{S}} P_{\mathrm{A}}$ and $C_{\mathrm{A}} P_{\mathrm{A}}$. 
Theoretical background. In order to understand the polarization properties of the half order resonant peaks, the tensor structure factor has to be calculated. The general form, based on the glide plane symmetry of the $\mathrm{SmC}_{\mathrm{S}} \mathrm{P}_{\mathrm{A}}$ structure and of the $2_{1}$ screw axis of the $\mathrm{SmC}_{\mathrm{A}} \mathrm{P}_{\mathrm{A}}$ phase is given by Dmitrienko in [11]. The calculation of the tensor elements was adapted to the bow shape in [35]:

$$
\begin{gathered}
\tilde{\tilde{F}}\left(\operatorname{Sm} C_{\mathrm{S}} P_{\mathrm{A}}\right)=\left[\begin{array}{ccc}
0 & i A \sin \alpha & 0 \\
i A \sin \alpha & 0 & i A \cos \alpha \\
0 & i A \cos \alpha & 0
\end{array}\right] \\
\tilde{\tilde{F}}\left(\operatorname{Sm} C_{\mathrm{A}} P_{\mathrm{A}}\right)=\left[\begin{array}{ccc}
0 & 0 & B \sin 2 \alpha \\
0 & 0 & i A \cos \alpha \\
B \sin 2 \alpha & i A \cos \alpha & 0
\end{array}\right]
\end{gathered}
$$

in which $\mathrm{A}$ and $\mathrm{B}$ are unknown complex parameters and $\alpha$ is the tilt angle of the string relative to the layer normal axis $\mathrm{Z}$.

The scattering amplitudes $\mathrm{A}_{\gamma \delta}$ derive simply from the tensors via the usual formulas [11]:

$$
\overline{\mathrm{A}}_{\gamma \delta}=\gamma \cdot \tilde{\tilde{F}} \delta
$$

in which the incident and diffracted polarization vectors $\delta$ and $\gamma$ can take the two values $\pi$ (in the scattering plane) or $\sigma$ (perpendicular to the scattering plane). The scattering geometry is shown in Fig. 11(a). In the experiments, the incident beam is always $\sigma$-polarized.

The polarization state of the resonant Bragg peaks depends on the azimuthal orientation $\varphi$ of the sample [11]. The general form of the scattered intensity can be found in $[11,35,36]$. The main features are as follows: in the $\mathrm{SmC}_{\mathrm{S}} \mathrm{P}_{\mathrm{A}}$ phase, the ratio of the two tensor elements is real and depends on the tilt angle $\alpha$ only (Eq. (2)). Consequently, the polarization of the resonant peaks is linear and its variation with the azimuth $\varphi$ can be exactly calculated with no free parameter if the tilt angle is known. In the $\mathrm{SmC}_{\mathrm{A}} \mathrm{P}_{\mathrm{A}}$ phase, this simplification does not occur, but the form of the tensor leads to a constanr $\pi$ polarization, independant of the azimuth $\varphi$. The theoretical behaviours are summarized in Eq. (4):

$$
\begin{aligned}
& \omega(\varphi)=\arctan \left[\left(\sin \theta_{B} \cos 2 \varphi-\cos \theta_{B} \cos \varphi / \tan \alpha\right) / \sin 2 \varphi\right] \text { for } \operatorname{SmC}_{\mathrm{S}} \mathrm{P}_{\mathrm{A}} \\
& \text { and } \\
& \omega(\varphi)=\pi / 2 \text { for } \operatorname{SmC}_{\mathrm{A}} \mathrm{P}_{\mathrm{A}}
\end{aligned}
$$

in which $\omega$ is the direction of the linear polarization of the resonant peaks relative to $\sigma$. It is plotted vs $\varphi$ in Fig. 19 .

Measuring the rotation of the polarization versus the azimuth $\varphi$ hence should enable a non ambiguous characterization of the structure of the $\mathrm{B}_{2}$ phases. The experimental results are shown in Figs. 20 and 21 for the $(16 \mathrm{OTBB})_{2}$ and $14 \mathrm{PBCl}$ compounds, respectively. In both cases, the alignment of the fluid liquid crystal is achieved by spreading a thin drop on a flat glass substrate. This method ensures an excellent alignment of the layers parallel to the substrate and a preferred in-plane orientation of the tilt along the direction of shear defined as $\varphi=0$.

Experiments on the $(16 \mathrm{OTBB})_{2}$ compound were carried out at sulphur K-edge. The data points are perfectly fitted to the $\mathrm{SmC}_{\mathrm{S}} \mathrm{P}_{\mathrm{A}}$ model, apart from a narrow region around $\varphi=90$ degrees (dashed line in Fig. 20). If an imperfect orientation of the liquid crystal is taken into account, the fit is excellent over the whole azimuthal range (solid line). The value of the tilt angle is obtained with a great accuracy ( $42.6 \pm 0.1$ degrees) in good agreement with the value obtained by other techniques [34]. 


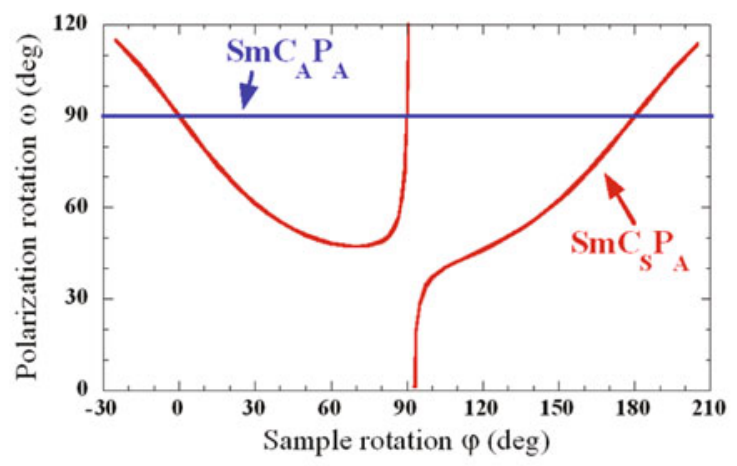

Fig. 19. Theoretical plot of the rotation $\omega$ of the linear polarization of the resonant Bragg peaks versus the azimuthal rotation of the sample in the two antiferroelectric models of the $\mathrm{B}_{2}$ phase (from Eq. (4)). The $\mathrm{SmC}_{\mathrm{S}} \mathrm{P}_{\mathrm{A}}$ curve is calculated for the value $\alpha=28$ degrees of the $14 \mathrm{PBCl}$ compound with no free parameter.

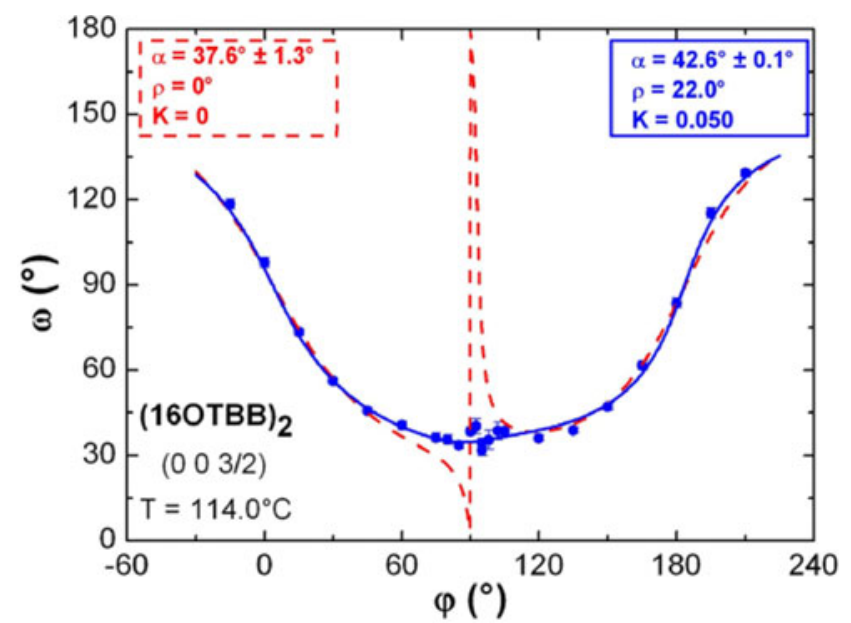

Fig. 20. Experimental plot of the rotation $\omega$ of the plane of polarization of the resonant $3 / 2$ Bragg peak vs sample rotation $\varphi$ for the (16OTBB) 2 compound. Dots are experimental points. The dashed line is a fit to the $\mathrm{SmC}_{\mathrm{S}} \mathrm{P}_{\mathrm{A}}$ model in the case of a perfect alignment. The solid line is a fit to the same model taking imperfect alignment into account [35].

Experiments on the $14 \mathrm{PBCl}$ compound, performed at chlorine K-edge, showed a different behaviour. The data points could neither be fitted to the $\mathrm{SmC}_{\mathrm{S}} \mathrm{P}_{\mathrm{A}}$ nor to the $\mathrm{SmC}_{\mathrm{A}} \mathrm{P}_{\mathrm{A}}$ model. If instead a coexistence of the two phases is assumed, the experimental data are well reproduced. Once again, the imperfect in-plane alignment of the biaxial sample has to be taken into account. The volume fraction of the homochiral $\mathrm{SmC}_{\mathrm{A}} \mathrm{P}_{\mathrm{A}}$ phase is extracted from the fit $f_{\mathrm{Vol}}=34 \pm 6 \%$.

These experiments have enabled the characterization of two of the four structures proposed for the $\mathrm{B}_{2}$ phase. The achiral $\mathrm{SmC}_{\mathrm{S}} \mathrm{P}_{\mathrm{A}}$ structure is the most frequent. Surprisingly, a homochiral phase $\left(\mathrm{SmC}_{\mathrm{A}} \mathrm{P}_{\mathrm{A}}\right)$ develops in an achiral material. This spontaneous chiral symmetry breaking is similar to the up-down symmetry breaking in Ising ferromagnets. The global achiral symmetry is saved by the formation of domains of opposite handedness. The homochiral phase is found to coexist with another structure, which is in principle forbidden by the phase rule in a pure compound. Such 


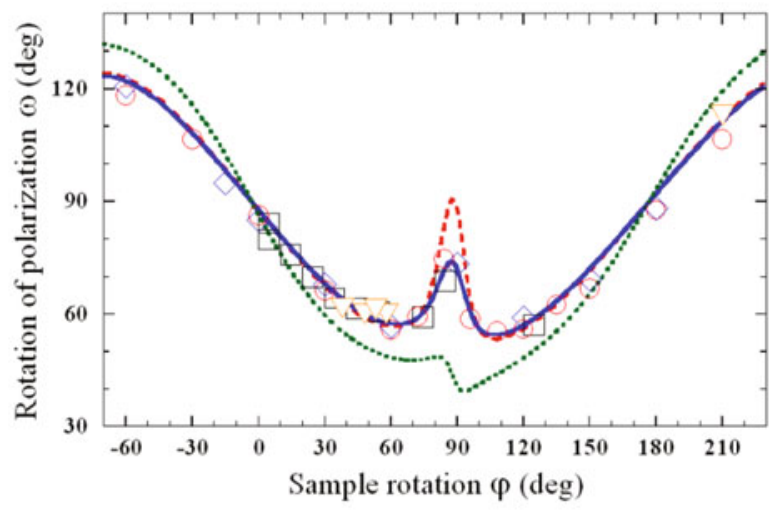

Fig. 21. Same plot as Fig. 20 for the $14 \mathrm{PBCl}$ compound. Different symbols correspond to different samples. The dashed and solid lines are the best fits to the model of coexisting $\mathrm{SmC}_{\mathrm{S}} \mathrm{P}_{\mathrm{A}}$ and $\mathrm{SmC}_{\mathrm{A}} \mathrm{P}_{\mathrm{A}}$ phases with a perfect in-plane alignment (dashed) and with a Gaussian distribution of in-plane orientation of width $15 \pm 1$ degree (solid). The dotted line is the polarization calculated for the pure $\mathrm{SmC}_{\mathrm{S}} \mathrm{P}_{\mathrm{A}}$ phase with the same distribution of in-plane orientation and no other free parameter [36].

coexistence may nevertheless occur in thin films if the interaction with the surfaces stabilizes a second phase or if metastable states are trapped by slow kinetics.

\section{Conclusion}

X-ray resonant scattering has proved to be a powerful tool to investigate the structure of liquid crystals. The restoration of forbidden reflexions by the resonant enhancement of the anisotropic tensor structure factor provides the experimentalists with new and highly important structural data. The technique has been indeed successfully used to solve important issues that had remained controversial for more than a decade. The structures of the antiferroelectric smectics and their related subphases have been elucidated with a high level of detail and theoretical studies have been strongly stimulated. The use of polarization analysis for structural studies in soft condensed matter is, to our knowledge, unprecedented.

It is also interesting to note that despite their fluid nature and their imperfect alignment, liquid crystals support fine and accurate experimental studies of their tensor structure factor.

Only dipolar interaction has been invoked so far. It is not clear yet if quadrupolar interaction is important and may bring additional structural information.

Future work will undoubtedly address the characterization of new subphases predicted by the most recent theories and the characterization of other phases appearing in bent-core systems [37]. The use of micro-focused X-ray beams also presents new opportunities to do resonant studies of micron-sized ordered domains of the biaxial bent-core systems [38], which prove difficult to uniformly align over longer length scales. Finally, there are new beamlines, including the SIRIUS beamline recently commissioned at the SOLEIL synchrotron and the ISR beamline planned for the new NSLS-II synchrotron, that cover the relevant edges for soft matter while providing full polarization control of the incident X-ray beam. Full polarization control will enable the unambiguous identification of the biaxial phase structures including their absolute sense of their chirality [39]. 
Special thanks and gratitude are due to Dr. Anne-Marie Levelut who initiated the resonant scattering studies in liquid crystals. PB thanks the "Formation Permanente" action of CNRS for financial support at the REXS 2011 Conference in Aussois.

\section{References}

1. P.G. de Gennes, J. Prost, The Physics of Liquid Crystals, 2nd edn. (Oxford University Press, 1993)

2. H.F. Gleeson, L.S. Hirst, Chem. Phys. Chem. 7, 321 (2006)

3. R.A. Reddy, C. Tschierske, J. Mater. Chem. 16, 907 (2006)

4. A.D.L. Chandani, E. Gorecka, Y. Ouchi, H. Takezoe, A. Fukuda, Jpn. Appl. Phys., Part 2, 28, L1265 (1989)

5. A. Fukuda, Y. Takanishi, T. Isozaki, K. Ishikawa, H. Takezoe, J. Mater. Chem. 4, 997 (1994)

6. Y. Galerne, L. Liébert, Phys. Rev. Lett. 64, 906 (1990)

7. P. Mach, R. Pindak, A.-M. Levelut, P. Barois, H.T. Nguyen, C.C. Huang, L. Furenlid, Phys. Rev. Lett. 81, 1015 (1998)

8. P. Mach, R. Pindak, A.-M. Levelut, P. Barois, H.T. Nguyen, H. Baltes, M. Hird, K. Toyne, A. Seed, J.W. Goodby, C.C. Huang, L. Furenlid, Phys. Rev. E 60, 6793 (1999)

9. D.H. Templeton, L.K. Templeton, Acta Cryst. A36, 237 (1980)

10. J.L. Hodeau, V. Favre-Nicolin, S. Bos, H. Renevier, E. Lorenzo, J.F. Berar, Chem. Rev. 101, 1843 (2001)

11. V.E. Dmitrienko, Acta Cryst. A39, 29 (1983)

12. A.-M. Levelut, B. Pansu, Phys. Rev. E 60, 6803 (1999)

13. A. Cady, J.A. Pitney, R. Pindak, L.S. Matkin, S.J. Watson, H.F. Gleeson, P. Cluzeau, P. Barois, A.-M. Levelut, W. Caliebe, J.W. Goodby, M. Hird, C.C. Huang, Phys. Rev. E 64, 050702(R) (2001)

14. P. Cluzeau, P. Gisse, V. Ravaine, A.M. Levelut, P. Barois, C.C. Huang, F. Rieutord, H.T. Nguyen, Ferroelectrics 244, 1 (2000)

15. N.W. Roberts, S. Jaradat, L.S. Hirst, M.S. Thurlow, Y. Wang, S.T. Wang, Z.Q. Liu, C.C. Huang, J. Bai, R. Pindak, H.F. Gleeson, Europhys. Lett. 72, 976 (2005)

16. P.D. Brimicombe, N.W. Roberts, S. Jaradat, C. Southern, S.-T. Wang, C.-C. Huang, E. DiMasi, R. Pindak, H.F. Gleeson, Eur. Phys. J. E 23, 281 (2007)

17. P.M. Johnson, D.A. Olson, S. Pankratz, T. Nguyen, J. Goodby, M. Hird, C.C. Huang, Phys. Rev. Lett. 84, 8470 (2000)

18. L.S. Hirst, S.J. Watson, H.F. Gleeson, P. Cluzeau, P. Barois, R. Pindak, J. Pitney, A. Cady, P.M. Johnson, C.C. Huang, A.-M. Levelut, G. Srajer, J. Pollmann, W. Caliebe, A. Seed, M.R. Herbert, J.W. Goodby, M. Hird, Phys. Rev. E 65, 041705 (2002)

19. Z.Q. Liu, S.T. Wang, B.K. McCoy, A. Cady, R. Pindak, W. Caliebe, K. Takekoshi, K. Ema, H.T. Nguyen, C.C. Huang, Phys. Rev. E 74, 030702(R) (2006)

20. L.S. Matkin, S.J. Watson, H.F. Gleeson, R. Pindak, J. Pitney, P.M. Johnson, C.C. Huang, P. Barois, A.M. Levelut, G. Srajer, J. Pollmann, J.W. Goodby, M. Hird, Phys. Rev. E 64, 021705 (2001)

21. J.T. Mills, H.F. Gleeson, J.W. Goodby, M. Hird, A. Seed, P. Styring, J. Matter. Chem. 8, 2385 (1998)

22. S. Jaradat, H.F. Gleeson, N. Roberts, Y. Wang, J. Mat. Chem. 16, 3753 (2006)

23. S. Jaradat, P. Brimicombe, C. Southern, S. Siemianowski, R. Pindak, E. DiMasi, M. Osipov, H.F. Gleeson, Phys. Rev. E 77, 010701(R) (2008)

24. S. Jaradat, M. Osipov, R. Pindak, H.F. Gleeson, Appl. Phys. Lett. 98043501 (2011)

25. P. Fernandes, P. Barois, E. Grelet, F. Nallet, J.W. Goodby, M. Hird, J.-S. Micha, Eur. Phys. J. E 20, 81 (2006)

26. M. Cepic, B. Zeks, Phys. Rev. Lett. 87, 085501 (2001)

27. D.A. Olson, X.F. Han, A. Cady, C.C. Huang, Phys. Rev. E 66, 021702 (2002)

28. P.V. Dolganov, V.M. Zhilin, V.K. Dolganov, E.I. Kats, Phys. Rev. E 67, 041716 (2003)

29. A.V. Emelyanenko, M.A. Osipov, Phys. Rev. E 68, 051703 (2003) 
30. M.B. Hamaneh, P.L. Taylor, Phys. Rev. E 72, 021706 (2005)

31. Shun Wang, LiDong Pan, R. Pindak, Z.Q. Liu, H.T. Nguyen, C.C. Huang, Phys. Rev. Lett. 104, 027801 (2010)

32. D. Link, G. Natale, R. Shao, J.E. Maclennan, N.A. Clark, E. Körblova, D.M. Walba, Science 278, 1924 (1997)

33. A. Cady, R. Pindak, W. Caliebe, P. Barois, W. Weissflog, H.T. Nguyen, C.C. Huang, Liq. Cryst. 29, 1101 (2002)

34. J.C. Rouillon, J.P. Marcerou, M. Laguerre, H.T. Nguyen, M.F. Achard, J. Mater. Chem. 11, $2946(2001)$

35. P. Fernandes, P. Barois, S.T. Wang, Z.Q. Liu, B.K. McCoy, C.C. Huang, R. Pindak, W. Caliebe, H.T. Nguyen, Phys. Rev. Lett. 99, 227801 (2007)

36. V. Ponsinet, P. Barois, LiDong Pan, Shun Wang, C.C. Huang, S.T. Wang, R. Pindak, U. Baumeister, W. Weissflog, Phys. Rev. E 84, 011706 (2011)

37. C.L. Folcia, J. Ortega, J. Etxebarria, LiDong Pan, Shun Wang, C.C. Huang, V. Ponsinet, P. Barois, R. Pindak, N. Gimeno, Phys. Rev. E 84, 010701(R) (2011)

38. Y. Takanishi, Y. Ohtsuka, Y. Takahashi, A. Iida, Phys. Rev. E 81, 011701 (2010)

39. Y. Tanaka, T. Takeuchi, S.W. Lovesey, K.S. Knight, A. Chainani, Y. Takata, M. Oura, Y. Senba, H. Ohashi, S. Shin, Phys. Rev. Lett. 100, 145502 (2008) 